\title{
Study on equivalent static method for the analysis of fatigue behavior of reinforced concrete beam
}

\author{
Fangping $\mathrm{Liu}^{1}$, Chen $\mathrm{Yu}^{1}$, Wentao $\mathrm{Yi}^{1}$ \\ ${ }^{1}$ School of Civil Engineering, Chongqing Three Gorges University, Chongqing 404020, China \\ Correspondence should be addressed to Fangping Liu;
}

\begin{abstract}
In order to analyze the whole process of fatigue behaviours of reinforced concrete beams, an equivalent static analysis method is proposed in this paper. Firstly, the constitutive models based on the degradation of stiffness and strength and the accumulation of residual strain of concrete and reinforcement subjected to fatigue loadings are deduced and established by coupling the uniaxial constitutive model of concrete and the ideal elastic-plastic model of reinforcement under static load. Secondly, based on the similarity of concrete failure under static load and fatigue load, the equivalent relationship between fatigue and static analysis can be constructed by using the concrete residual strain as the equivalent parameter. On this basis, an equivalent static method for the analysis of fatigue behavior of reinforced concrete beam is proposed. At last, three reinforced concrete beams with rectangular section are tested, and the fatigue behaviours are analyzed by the method proposed in this paper. The numerical simulation results are compared with the field test data. The result shows that the numerical simulation results are in good agreement with the experimental results, which verifies the reliability and practicability of the method.
\end{abstract}

\section{Fatigue Constitutive Model}

\subsection{Concrete}

1.1.1 Fatigue stiffness. Under fatigue load, the change of elastic modulus of concrete is an important index to measure the degradation of stiffness. The research shows that the elastic modulus of concrete changes in three stages with the fatigue times: In the first stage, it decays rapidly; in the second stage, it shows stable linear degradation, which accounts for $75 \% \sim 80 \%$ of the fatigue life; in the third stage, it decays rapidly and unsteadily until the fatigue failure [1]. According to a large number of experimental studies in reference [2], the relationship between the elastic modulus and the fatigue times of the concrete in second stage is as follows:

$$
\begin{gathered}
E_{N}=\left(1-0.33 N / N_{\mathrm{f}}\right) E_{0} \\
\lg N_{f}=14.7-13.5\left[\left(\sigma_{\max }-\sigma_{\min }\right) /\left(f_{c u}-\sigma_{\min }\right)\right]
\end{gathered}
$$

In formula $(1) \sim(2): E_{0}$ is the initial elastic modulus of concrete; $E_{N}$ is the elastic modulus of concrete after $N$ times of fatigue; $N_{f}$ is the fatigue life [3], which is used according to the literature [4]; $\sigma_{\max }$ and $\sigma_{\min }$ are the initial instantaneous stress under the upper and lower limit load respectively; $f_{c u}$ is the compressive strength of concrete cube.
1.1.2 Fatigue strength. A large number of studies show that for a certain number of fatigue cycles, the fatigue strength depends on the maximum stress and the minimum stress in the cycle. According to the research results in reference [5], the relationship between the fatigue times $N$ and the upper limit of compressive stress $\sigma_{c, \max }$ (i.e. residual fatigue compressive strength $f_{c N}$ ) and the lower limit of compressive stress $\sigma_{c, \text { min }}$ is as follows:

$$
\lg N=\frac{1}{\beta}\left[\frac{1-\sigma_{c, \max } / f_{c u}}{1-\sigma_{c, \min } / \sigma_{c, \max }}\right]
$$

In formula (3): $\beta$ is the material constant, the value range is $0.064 \sim 0.080$, which can be obtained by regression analysis.

The relationship between the fatigue times $N$ and the upper limit of tensile stress $\sigma_{t, \max }$ (i.e. residual fatigue tensile strength $f_{t N}$ ) and the lower limit of tensile stress $\sigma_{t, \min }$ is as follows:

$$
\lg N=\frac{1}{\beta}\left[\frac{\left(\sigma_{t, \text { max }}-\sigma_{t, \text { min }}\right) / f_{t}}{1-\sigma_{t, \text { min }} / f_{t}}\right]
$$

In formula (4): $f_{t}$ is the static tensile strength of concrete. When $f_{c N}$ and $f_{t N}$ are determined, according to table C.2.4 in specification [6], the peak compressive strain $\varepsilon_{c r}$ and peak tensile strain $\varepsilon_{t r}$ can be obtained. 
1.1.3 Fatigue residual strain. In the whole fatigue process of concrete, with the increase of fatigue times, the elastic modulus is degenerating, the residual strength is degenerating, and the residual strain is increasing. The experimental results show that the residual strain also changes in three stages along with the increase of fatigue times [7]. According to the research results of literature [8], the change of fatigue residual strain is as follows:

$$
\varepsilon_{f, r}(N)=\varepsilon_{f, r}(1)+\left(k_{1} \varepsilon_{\max }^{k_{2}}\left(1-\varepsilon_{\min } / \varepsilon_{\max }\right)^{k_{3}}\right) N^{k_{4}} / \varepsilon_{k}^{k_{s}}
$$

In formula $(5): \varepsilon_{f, r}(N)$ is the residual strain after $N$ times of fatigue; $\varepsilon_{\max }$ and $\varepsilon_{\min }$ are the initial instantaneous strains under the upper and lower limit load respectively. ${ }^{\varepsilon_{f, r}}(1)$ is the residual strain caused by the first cycle, $\varepsilon_{f, r}(1)=0.25\left(\varepsilon_{\max } / \varepsilon_{k}\right)^{2}, \quad k_{1}, k_{2}, k_{3}, k_{4}, k_{5}$ are the material constant, which can be obtained by regression analysis of test data. $\varepsilon_{k}$ is the total longitudinal strain at the beginning of the third stage, and its value can be replaced by the peak failure strain $\varepsilon_{\text {unsab }}$ [9-10].

1.1.4 Fatigue constitutive model. Based on above analysis, the compressive constitutive models based on the degradation of stiffness and strength and the accumulation of residual strain of concrete subjected to fatigue loadings is established by coupling the uniaxial constitutive model under static load [11]. In this process, the hysteresis between unloading and reloading curves is ignored. The model equations are as follows $(6) \sim(10)$ :

$$
\begin{gathered}
\sigma_{r, c}(N)=k_{c} E_{N}\left(\varepsilon-\varepsilon_{f, r}(N)\right) \\
k_{c}=\left\{\begin{array}{c}
\frac{\rho_{c} n}{n-1+x_{c}^{n}}, x_{c} \leq 1 \\
\frac{\rho_{c}}{\alpha_{c}\left(x_{c}-1\right)^{2}+x_{c}}, x_{c}>1 \\
\rho_{c}=f_{c N} /\left(E_{N} \varepsilon_{c r}\right) \\
x_{c}=\left(\varepsilon-\varepsilon_{f, r}(N)\right) / \varepsilon_{c r} \\
n=E_{N} \varepsilon_{c r} / f_{c N}
\end{array}\right.
\end{gathered}
$$

Similarly, the tensile fatigue constitutive model equations are as follows (11) (14)

$$
\begin{gathered}
\sigma_{r, t}(N)=k_{t} E_{N}\left(\varepsilon-\varepsilon_{f, r}(N)\right) \\
k_{t}=\left\{\begin{array}{l}
\rho_{t}\left(1.2-0.2 x_{t}^{5}\right), x_{t} \leq 1 \\
\frac{\rho_{t}}{\alpha_{t}\left(x_{t}-1\right)^{1.7}+x_{t}}, x_{t}>1
\end{array}\right. \\
\rho_{t}=\left(\varepsilon-\varepsilon_{t N} / E_{N} \varepsilon_{t r}(N)\right) / \varepsilon_{t r}
\end{gathered}
$$

In formula $(6) \sim(14): \sigma_{r, c}(N)$ and $\sigma_{r, t}(N)$ are the compressive and tensile stresses after $N$ times of fatigue; $\varepsilon$ is the strain after $N$ times of fatigue; $k_{c}$ and $k_{t}$ are damage evolution parameters under compression and tension fatigue; $\alpha_{c}$ and $\alpha_{t}$ are the shape factor of the descend phase in stress-strain curve, which can be taken from table C.2.4 in reference [6]; ${ }^{\varepsilon_{f, r}}(N)$ is the residual strain after the $N$ times loading and unloading; $E_{N}$ is the elastic modulus after $N$ times of fatigue; $f_{c N}$ and $f_{t N}$ are the residual compressive and tensile strength after $N$ times of fatigue; $\varepsilon_{c r}$ and $\varepsilon_{t r}$ are the peak compressive strain and the peak tensile strain under the Nth time loading.

\subsection{Reinforced Rebar}

Through the fatigue test of reinforced rebar, it is found that most of the deformation is in the elastic stage in the process of fatigue loading. So it can be assumed that the elastic modulus does not degrade in the whole process [10], and the residual strain can be ignored. The fatigue failure of the reinforced rebar is the instantaneous fracture of the section. The main reason is that the residual effective cross-sectional area can not bear the tensile load.

According to the research results of the author's doctoral dissertation [12], the tensile constitutive model based on the degradation of strength and the accumulation of residual strain of reinforced rebar subjected to fatigue loadings is established by coupling the ideal elastic-plastic model under static load. The model equations are as follows $(15) \sim(18)$ :

$$
\begin{gathered}
\sigma_{s}(N)= \begin{cases}\mathrm{E}_{\mathrm{s}} \varepsilon_{\mathrm{s}}(N) & \Delta \varepsilon_{\mathrm{r}}(N-1)<\varepsilon_{\mathrm{s}}(N) \leq \varepsilon_{\mathrm{y}}(N) \\
f_{\mathrm{y}}(N) & \varepsilon_{\mathrm{s}}(N)>\varepsilon_{\mathrm{y}}(N)\end{cases} \\
f_{\mathrm{y}}(N)=f_{\mathrm{y}}\left[1-\left(1-\frac{\sigma_{\max }\left(N_{f}\right)}{f_{\mathrm{y}}}\right) \cdot\left(\frac{N}{N_{f}}\right)^{c}\right] \\
\Delta \varepsilon_{\mathrm{r}}(N-1)=\left[f_{\mathrm{y}}(N)-f_{\mathrm{y}}(N-1)\right] / \mathrm{E}_{\mathrm{s}} \\
\varepsilon_{\mathrm{y}}(N)=\Delta \varepsilon_{\mathrm{r}}(N-1)+f_{\mathrm{y}} / \mathrm{E}_{\mathrm{s}}
\end{gathered}
$$

In formula $(15) \sim(18): \sigma_{s}(N)$ and $\varepsilon_{s}(N)$ are the stress and strain at loading times $N ; f_{y}(N)$ and $\varepsilon_{y}(N)$ are the yield stress and strain at loading times $N ; \Delta \varepsilon_{\mathrm{r}}(N-1)$ is the residual strain at loading times $N-1 ; \mathrm{E}_{\mathrm{s}}$ is the initial elastic modulus; $f_{y}$ is the initial yield strength.

\section{Analytical Method}

\subsection{Equivalent Parameter and Equivalent State}

2.1.1 Equivalent parameter. The assumption of concrete deformation uniqueness is proposed by SINHA [13]. The assumption is that no matter what the previous repeated load history is, the relationship between load and deformation will remain unchanged with the same repeated load as long as the residual deformation is the same. This is independent of the previous load deformation history. The hypothesis has been proved by many experiments. It is also proved in literature $[6,7]$ that the residual strain reflects the micro plastic deformation and the degree of micro crack irrecoverability of concrete, so it can better reflect the damage characteristics of material itself than other parameters. Therefore, the following assumptions are made in this paper: after one time of static (load $=P_{1}, P_{1}>P_{\max }$ ) loading and unloading, the concrete beam is in state 1(corresponding path-A in figure 1), and the residual compressive strain of concrete 
at the compression edge is $\varepsilon_{r}$; meanwhile after $N$ times of fatigue load(the load range is $P_{\min } \sim P_{\max }$ ), the same beam is in state 2 (corresponding path-B in figure 1 ), and the residual compressive strain of concrete at the compression edge is $\varepsilon_{f, r}$ (see figure 1); if the residual strain of the two is equal, that is $\varepsilon_{r}={ }^{\varepsilon_{f, r}}$, then it can be considered that the energy dissipation and the concrete damage in state 1 is the same as that of state 2 .

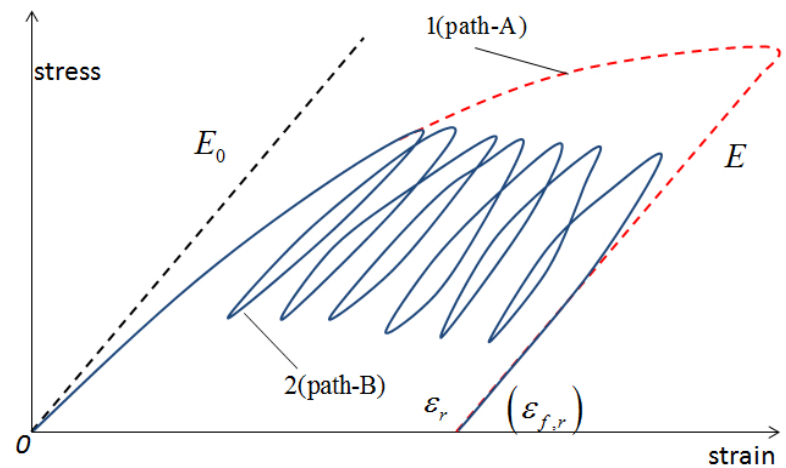

Figure 1. Diagram of equivalent state

If the bond degradation and relative slip between reinforcement and concrete are not considered, the strain of reinforcement is equal to that of concrete in the same position, so the strain of reinforcement in state 1 is equal to state 2 , the stress is also the same. Therefore, the residual strain of concrete in the compression zone can be taken as an equivalent parameter to establish the equivalent state of fatigue loading and static loading. Then, the state 2 beam which has experienced $N$ times of fatigue load $\left(P_{\min } \sim P_{\max }\right)$ can be equivalent to the state 1 which has experienced one time static loading-unloading $\left(\operatorname{load}=P_{1}\right)$. If the equivalent static analysis is carried out with different fatigue times according to the characteristics of each stage, the development of deformations, strains and stresses of concrete structures after any fatigue times can be obtained.

2.1.2 Equivalent state. It can be seen from figure 1 that the residual strain of concrete under static loading can be obtained from equations (19) - (21):

$$
\begin{aligned}
& \varepsilon_{r}=\varepsilon-\varepsilon_{e}=\varepsilon-\sigma / E \\
& E=(1-d) E_{0} \\
& d=1-\sqrt{\sigma /\left(\varepsilon \cdot E_{0}\right)}
\end{aligned}
$$

In formula (19) (21): $\varepsilon, \varepsilon_{e}$ and $\varepsilon_{r}$ are the total strain, elastic strain and residual strain of concrete at any time; $E$ is the unloading elastic modulus of concrete at any time (as shown in figure 1); $E_{0}$ is the initial elastic modulus; $d$ is the damage factor.

In conclusion, based on the above concrete fatigue constitutive model, from equation $(19) \sim(21)$, the residual strain of concrete at any time can be obtained by static loading. According to formula (5), there is $\varepsilon_{r}=\varepsilon_{f, r}$. Therefore, the equivalent static loading-unloading path $\mathrm{A}$ can be established from the fatigue loading path B by concrete residual strain.

\subsection{Fatigue Failure Criteria.}

2.2.1 Concrete. The original defects in the concrete continue to expand under fatigue load. The material behavior has degenerated seriously and can no longer be used, even if it does not reach the ultimate failure limit state. Research shows that when the residual strain of concrete reaches 0.4 times of the ultimate strain under fatigue load, the concrete has been seriously damaged and can no longer be used effectively. Consequently, it can be considered that the fatigue failure of concrete has occurred at this time, and the fatigue failure criterion of concrete under fatigue load is as follows:

$$
\varepsilon_{f, r} \geq 0.4 \varepsilon_{0}=0.4 f_{c} / E_{0}
$$

In formula (22), $f_{c}$ is the ultimate strength of concrete under static load; $\varepsilon_{0}$ is the strain corresponding to $f_{c}$.

2.2.2 Reinforced rebar. Brittle fracture is the sign of fatigue failure of reinforced rebar. Generally, Whether or not the stress amplitude of reinforcement exceeds the allowable value is regarded as the basis of its failure. The allowable stress amplitude can be determined by the S-N curve in specification [6].

\section{Experimental Verification}

\subsection{Experimental Overview}

In order to verify the equivalent static analysis method for the fatigue behavior of reinforced concrete beams proposed in the paper, three reinforced concrete rectangular section beams were fabricated in the laboratory. The concrete strength of the experimental beam is $\mathrm{C} 30$, and the longitudinal main reinforcement is HRB335 grade threaded bar with a diameter of $20 \mathrm{~mm}$. The beam section size is $200 \mathrm{~mm}$ (width) $\times 400 \mathrm{~mm}$ (height), the beam length is $3300 \mathrm{~mm}$ (clear span

\begin{tabular}{|c|c|c|c|}
\hline \multicolumn{2}{|c|}{ Beam number } & \multicolumn{2}{|c|}{ Loading System $/ k N$} \\
\hline \multirow[t]{2}{*}{ JL-1 } & & Static Load Te & \\
\hline & $\begin{array}{l}\text { Lower Limit } \\
\quad\left(P_{\min }\right)\end{array}$ & $\begin{array}{l}\text { Upper Limit } \\
\left(\Delta P_{\max }\right)\end{array}$ & $\begin{array}{c}\text { Amplitude }(\Delta \\
P)\end{array}$ \\
\hline JL-2 & $0.1 P_{u}$ & $0.6 P_{u}$ & $0.5 P_{u}$ \\
\hline JL-3 & $0.1 P_{u}$ & $0.7 P_{u}$ & $0.6 P_{u}$ \\
\hline
\end{tabular}
$3000 \mathrm{~mm}$ ). The loading condition is shown in table 1 .

Table1. loading condition of experimental beam $\left(P_{u}\right.$ is the ultimate load)

JL-1 beam is used for static load test to test the relationship between load and mid-span deflection and the ultimate load required for fatigue test. The static load test is carried out in the way of step loading, and the load increment is $20 \mathrm{kN}$. The fatigue tests of JL-2 and JL-3 beams under different stress levels are used to find the relationship between load and mid- span deflection as well as the strain of concrete and reinforcement. 


\subsection{Finite Element Model}

Based on the above analysis process, the finite element software ANSYS is used for numerical analysis of the experimental beam. In the modeling process, SOLID65 element is used to simulate the concrete, and link8 element is used to simulate the reinforced rebar. Due to the symmetry of structure and load, half of the structure can be used to describe the problem to be studied. The mapping grid method is adopted. figure 2 is the half finite element model of the structure after mesh generation.

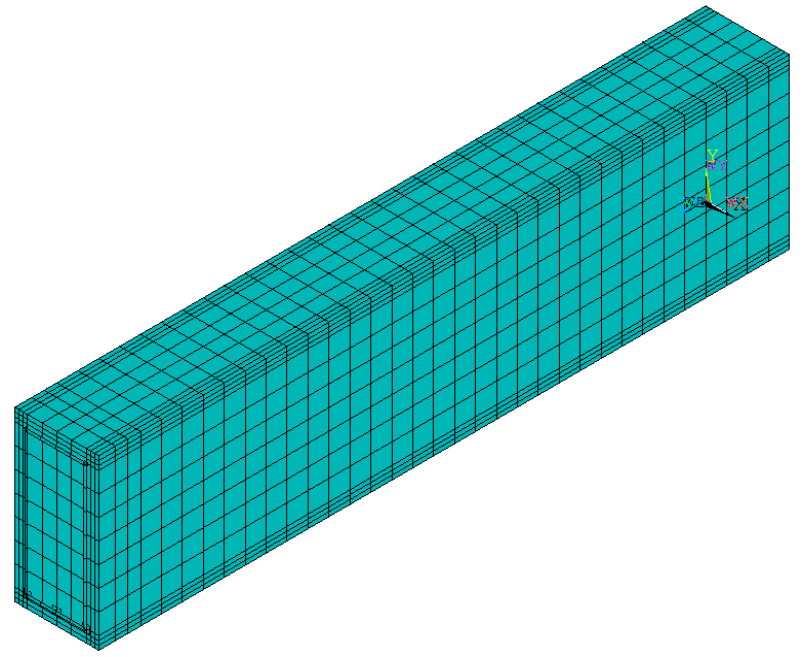

Figure 2. half finite element model

\subsection{Result and Discussion.}

\subsubsection{Mid-span deflection.}

The experimental and simulation curve of load and midspan deflection of JL-1 beam under static grading load are shown in Figure 3. It can be seen from the curve that the test results are slightly larger than the simulation results, the error between the two is not more than $10 \%$, and the change trend in the whole process is basically the same. All indicates that the simulation method and the selected parameters are reasonable. Finally, the experimental cracking load is $40 \mathrm{kN}$ and the ultimate load is $220 \mathrm{kN}$.

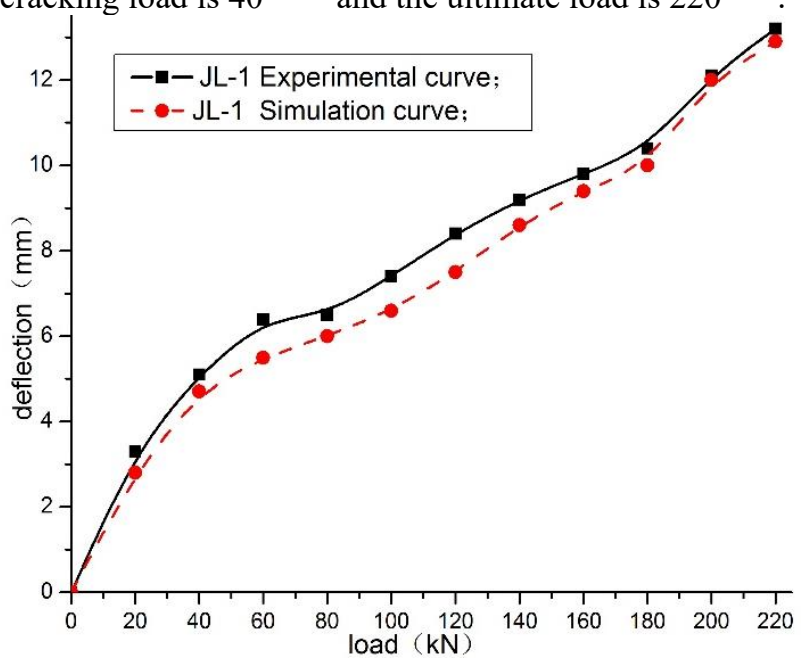

Figure 3. static load and mid-span deflection curve of JL-1 beam

When establishing the static equivalent state of JL-2 and JL-3 beams at 200000 times of fatigue loading, first try to take the equivalent static load and carry out one time loading-unloading, then modify the material constitutive relation to 200000 times of fatigue and carry out the static analysis, and the calculated values of load with corresponding deflection can be obtained finally. Similarly, the calculated values of load with corresponding deflection after 600000 times of fatigue and 1 million times of fatigue also can be obtained. The experimental and simulation curves of load with mid-span deflection of JL-2 and JL-3 beams are shown in figure 4-6.

It can be seen from figure 6-8 that the numerical simulation curve is in good agreement with the corresponding test curve. The relative error is generally within $10 \%$. Before the cracking load $40 \mathrm{kN}$, the structural stiffness is large. There is a turning point in the curve when the load reaches $40 \mathrm{kN}$, which shows that the stress redistribution occurs in the section after the concrete cracking, and the crack makes the structural stiffness significantly reduced. After a certain number of time fatigue loading, the number of cracks in the structure has been basically stable, and the section characteristics have changed from elastic-plastic to elastic. The slope of loaddeflection curve of one million times of fatigue and 600000 times of fatigue is slightly lower than that of 200000 times, but it is not significant, which indicates that the structural stiffness has been stabilized after 200000 times. In a word, the calculation results of fatigue damage on the structural stiffness are similar to the test results.

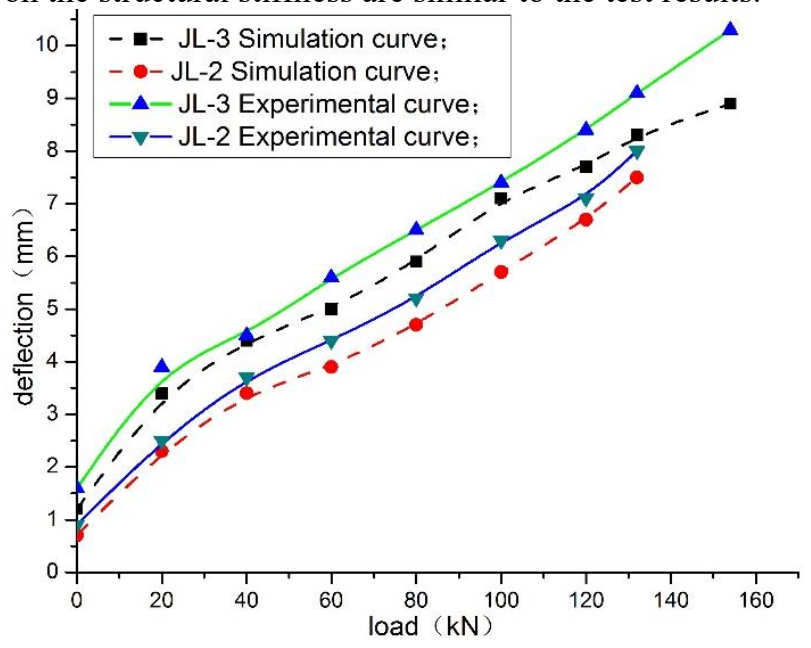

Figure 4. Test and simulation curves of load and mid-span deflection after 200000 times of fatigue 


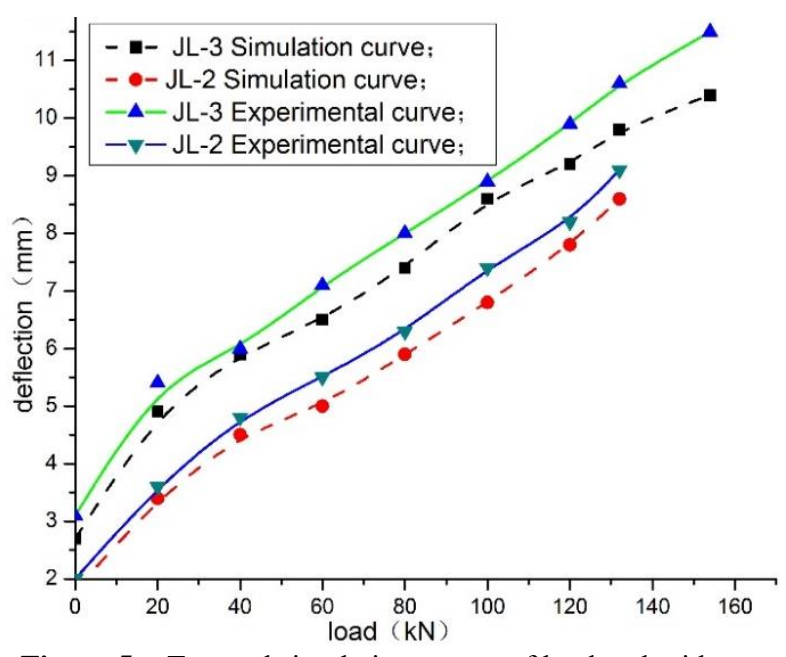

Figure 5. Test and simulation curves of load and mid-span

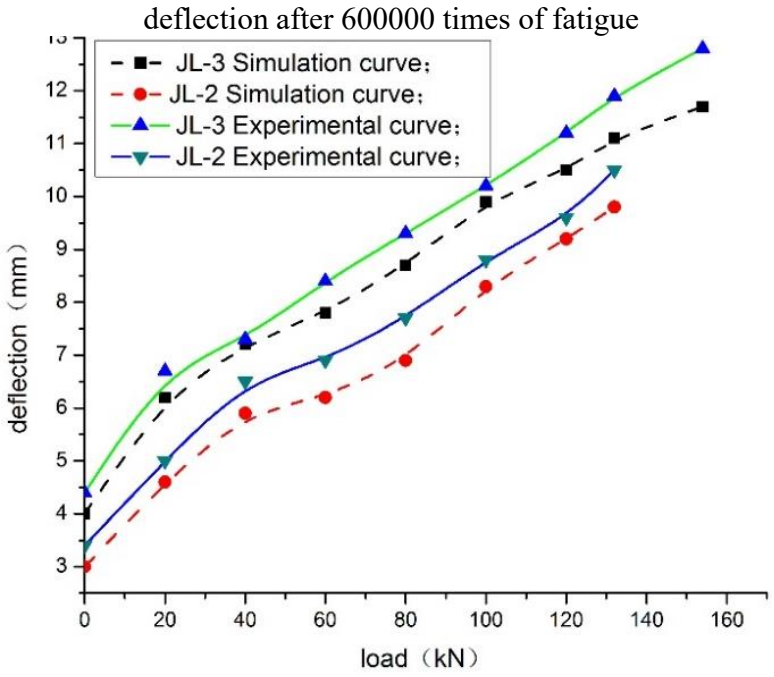

Figure 6. Test and simulation curves of load and mid-span deflection after 1000000 times of fatigue

3.3.2 Concrete compressive strain. In order to get the development process of concrete strain in compression zone under fatigue load, the equivalent static analysis of JL-2 and JL-3 beams are carried out by taking 200000 times as the loading interval. The minimum strains $\varepsilon_{\min }$ and the maximum strains $\varepsilon_{\max }$ in compression zone are shown in figure 7-8.

It can be seen from Figure 7-8 that before 200000 times, the minimum values $\varepsilon_{\text {min }}$ and the maximum values $\varepsilon_{\max }$ of the concrete increase relatively fast. The increasing trend gradually slows down after 200000 times, indicating that the fatigue strain gradually transits from the first stage to the second stage after 200000 times of fatigue. It also verifies the "three-stage deformation law" of concrete fatigue development. In the whole process, the minimum and the maximum strain of concrete gradually increase with the increase of fatigue number $N$, and the simulation value is slightly smaller than the experimental value, but the change rule is the same.

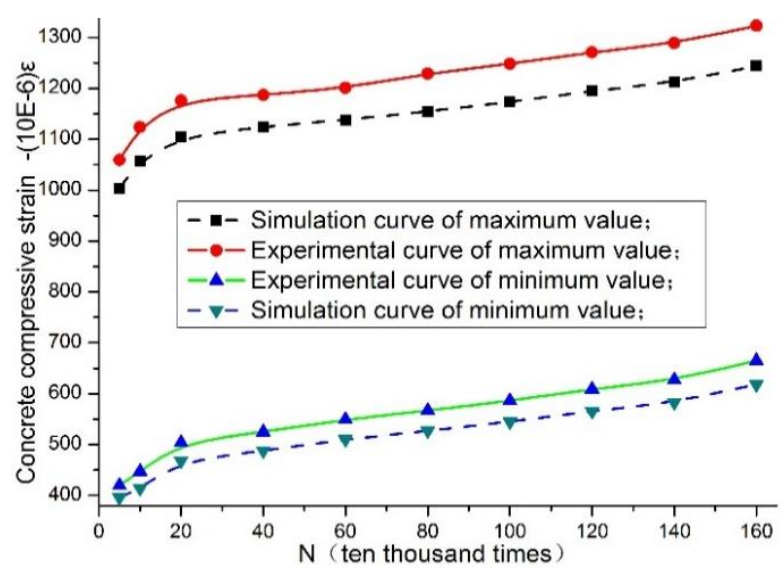

Figure 7. Concrete fatigue compressive strain of JL-2 beam

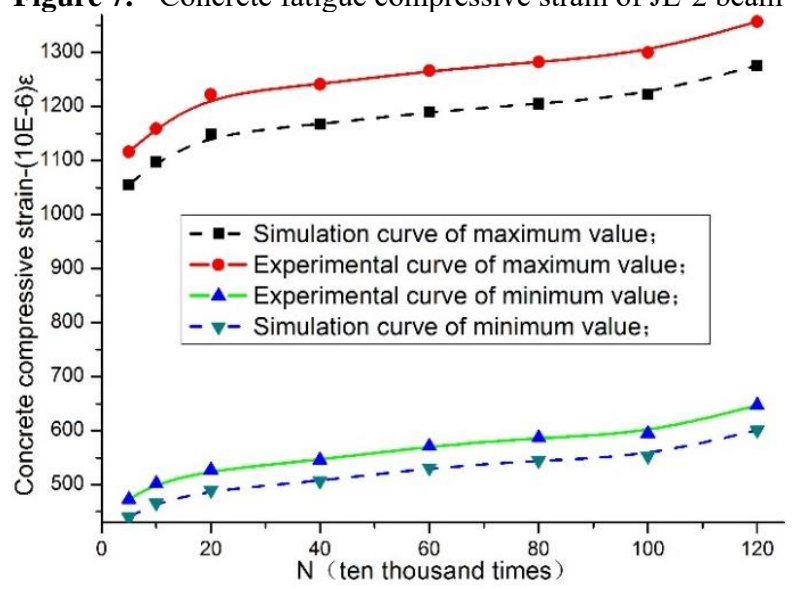

Figure 8. Concrete fatigue compressive strain of JL-3 beam

3.3.3 Reinforcement tensile strain.In order to get the development process of reinforcement tensile strain under fatigue load, the equivalent static analysis of JL-2 and JL3 beams are carried out by taking 200000 times as the loading interval. The minimum tensile strain $\varepsilon_{\min }$ and the maximum tensile strain $\varepsilon_{\max }$ are shown in figure 9-10.

From figure $9-10$, it can be seen that the minimum tensile strain $\varepsilon_{\min }$ and the maximum tensile strain $\varepsilon_{\max }$ of the reinforcement increase rapidly and obviously in the early stage. When the number of fatigue cycles exceeds 200000, the strain increment decreases, and then it enters a relatively stable growth stage. In general, the minimum and maximum tensile strain of reinforcement increase with the increase of fatigue number $N$, and the change rule of simulation result is consistent with that of experiment result.

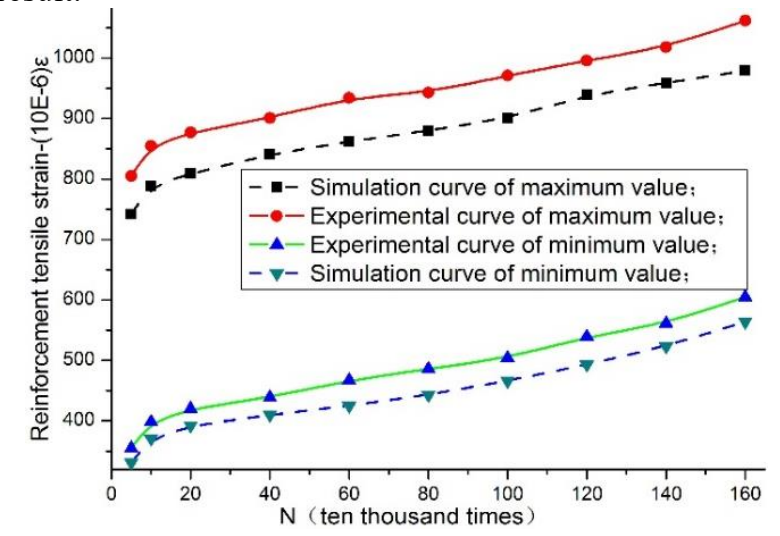




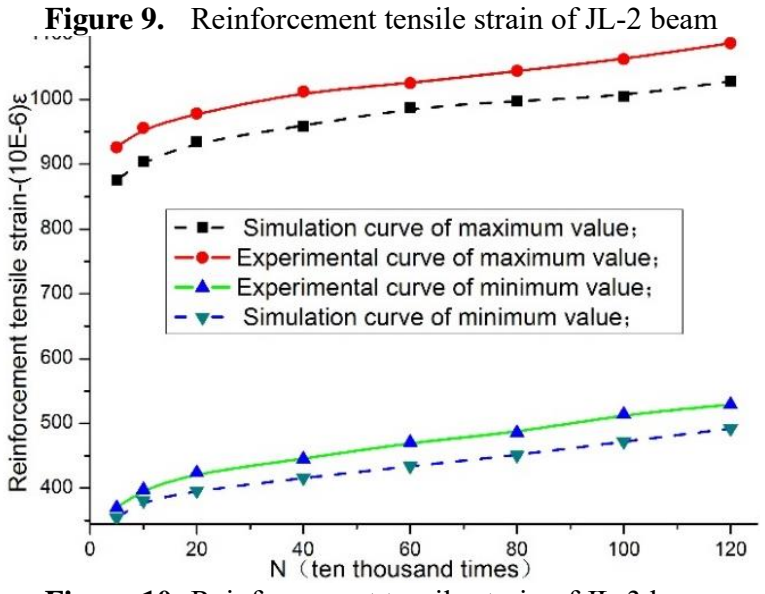

Figure 10. Reinforcement tensile strain of JL-3 beam

\section{Conclusion.}

(1) Through the evolution analysis of the fatigue stiffness, fatigue strength and fatigue residual strain of concrete and reinforcement materials, the fatigue constitutive models of concrete and reinforcement after any fatigue times are established based on the uniaxial constitutive model of concrete and the ideal elastic-plastic model of reinforcement under static load.

(2) Based on the similarity of concrete failure under static load and fatigue load, the equivalent relationship between fatigue and static analysis is constructed by using the concrete residual strain as the equivalent parameter. On the basis, the static method for the analysis of fatigue behavior of reinforced concrete beam is proposed.

(3) Three reinforced concrete beams with rectangular section are tested, and the corresponding fatigue behaviours are analyzed by the method proposed in the paper. The numerical simulation results are compared with the field test data. The result shows that the numerical simulation results are in good agreement with the experimental results, which verifies the reliability and practicability of the method.

\section{Acknowledgments}

This project was supported by the Project of Chongqing science and Technology Committee (cstc2018jcyjAX0360), Scientific and Technological Research Program of Chongqing Municipal Education Commission ( ( KJQN201901207) , Scientific and Technological Research Program of Chongqing Municipal Education Commission（KJQN202001204）.

\section{References}

1. S.Y. Wang, L. X. Zhang and R. B. Xu, "Deterioration laws of concrete elastic modulus under fatigue loading," Mechanics in Engineering, vol. 25, no. 5, pp. 55-57, 2003.

2. J. O. holmam, "Fatigue of concrete by constant and variable amplitude loading," Fatigue of Concrete Structure, vol. 75, no. 4, pp. 71-110, 1982.
3. The Concrete Fatigue Task Forces, "Research on failure reliability checking calculation method of reinforced concrete flexural components," China Construction Industry Publishing House, Beijing, China, pp.26-27,1994.

4. X. F. Feng, "Study on fatigue behavior of P.P.C beams with mixed reinforcement," Dalian University of Technology, Dalian, China, 2005.

5. S. Teng and F Wang, "Finite element analysis of reinforced concrete deep beams under fatigue loading," ACI Structural Journal, vol. 98, no. 3, pp. 315-323, 2001.

6. National Standard of the People's Republic of China, "GB50010-2010 Code for design of concrete structures,": China Building Industry Press, Beijing, 2002.

7. C.Y. Li, Y. P. Su and G. F. Zhao, "Study ofresidual strain of concrete under fatigue loading," Journal of Dalian University of Technology, vol. 41, no. 3, pp. 355-358, 2001.

8. X. H. Meng, "Experimental and theoretical research on residual strength of concrete under fatigue loading," Dalian University of Technology, Dalian, China, 2006.

9. K. T. Gyll, "Fracture mechanics models for fatigue in concrete structures," University of Technology, Lulea, Sweden, 1983.

10. W. Qian, D. P. Qi and W. C. Xue, "Full-range analysis on behaviors of concrete beams prestressed with cfrp tendons under fatigue load cycles," Journal of Vibration and Shock, vol. 17, no. 5, pp. 125-129, 2008.

11. J.S. Zhu, Y. P. Song and R. C. Xiao, "Fatigue behavior and post-damaged equivalent constitutive law of plain concrete," Journal of Building Materials, vol. 8, no. 6, pp. 609-614, 2005.

12. F. P. Liu, "Research on Residual Bearing Capacity of Reinforced Concrete Beams After Fatigue Loads," Chongqing Jiaotong University, Chongqing, China, 2016.

13. B. P. Sinha, K. H. Gasrtic and L. G. Tulni, "Stress-strain relations for concrete under cyclic loading," ACI Journal, vol. 61, no. 2, pp. 195-211, 1964. 